European Phys. Lett., 80, (2007), 44001.

A. G. Ramm, "Scattering by many small bodies and applications to condensed matter physics", European Phys. Lett., 80, (2007), 44001. 


\title{
Scattering by many small bodies and applications to condensed matter physics
}

\author{
A. G. Ramm \\ (Mathematics Department, Kansas St. University, \\ Manhattan, KS66506, USA \\ and TU Darmstadt, Germany) \\ ramm@math.KSU.edu
}

PACS: 03.04.Kf, 43.20.tg, 62.30.dt

Key words: many-body scattering, scattering by small particles, metamaterials

\begin{abstract}
Equations for the effective field in the medium, in which many small particles are embedded, are derived. It is shown how to create a material with a desired refraction coefficient by embedding in a given material many small particles.
\end{abstract}

\section{Introduction and statement of the results}

Let $D \subset \mathbb{R}^{3}$ be a bounded domain with a boundary $S$, filled with a material whose refraction coefficient $n_{0}^{2}(x)$ is known, $x \in \mathbb{R}^{3}$. The scattering problem consists of solving the equation:

$$
L_{0} u_{0}:=\left[\nabla^{2}+k^{2} n_{0}^{2}(x)\right] u_{0}=0 \quad \text { in } \mathbb{R}^{3}, \quad u_{0}=e^{i k \alpha \cdot x}+v_{0},
$$

where $k>0$ is fixed, $\alpha \in S^{2}$ is a unit vector in the direction of the incident wave, $n_{0}^{2}=1$ in $D^{\prime}=\mathbb{R}^{3} \backslash D, v_{0}$ is the scattered field satisfying the radiation condition:

$$
\partial v_{0} / \partial r-i k v_{0}=o\left(r^{-1}\right), \quad r:=|x| \rightarrow \infty .
$$

One can write $L_{0}=\nabla^{2}+k^{2}-q_{0}(x), q_{0}(x):=k^{2}\left[1-n_{0}^{2}(x)\right], q_{0}=0$ in $D^{\prime}$. The $k$-dependence in $q_{0}(x)$ is not shown because $k$ is fixed. Let $G(x, y)$ be the resolvent kernel of $L_{0}: L_{0} G=-\delta(x-y), \delta(x)$ is the delta-function, $G$ satisfies the radiation condition. Assume that $M \gg 1$ small particles $D_{m}$ are embedded into $D, a:=\frac{1}{2} \max _{1 \leq m \leq M} \operatorname{diam} D_{m}, n_{0} k a \ll 1, n_{0}=\max _{x \in \mathbb{R}^{3}}\left|n_{0}(x)\right|, d=$ : 
min $\operatorname{dist}_{m \neq j}\left(D_{m}, D_{j}\right), d \gg a$. Since $q_{0}(x)$ is known, we assume that $G(x, y)$ and $u_{0}(x)$ are known. Consider the scattering problem:

$$
\begin{aligned}
& L_{0} u_{M}=0 \quad \text { in } \mathbb{R}^{3} \backslash \bigcup_{m=1}^{M} D_{m}, \quad u_{M}=u_{0}+v_{M}, \\
& \frac{\partial u_{M}}{\partial N}=\zeta_{m} u_{M} \text { on } S_{m}, \quad 1 \leq m \leq M,
\end{aligned}
$$

where $S_{m}$ is the boundary of $D_{m}, N$ is the unit normal to $S_{m}$ pointing out of $D_{m}$, $\zeta_{m}$ is the boundary impedance, $v_{M}$ satisfies the radiation condition. We assume that $S_{m}$ are sufficiently smooth, e.g., $S_{m} \in C^{1, \lambda}, \lambda>0$, uniformly in $m$, and

$$
\operatorname{Im} q_{0}(x) \leq 0, \quad \operatorname{Im} \zeta_{m} \leq 0
$$

One can prove that problem (3), (4) has a unique solution under these assumptions, and this solution is of the form:

$$
u_{M}=u_{0}(x)+\sum_{m=1}^{M} \int_{S_{m}} G(x, s) \sigma_{m}(s) d s
$$

where the functions $\sigma_{m}$ are to be chosen so that conditions (4) are satisfied. Let $Q_{m}:=\int_{S_{m}} \sigma_{m}(s) d s$. Write (6) as

$$
u_{M}=u_{0}+\sum_{m=1}^{M} G\left(x, x_{m}\right) Q_{m}+\sum_{m=1}^{M} \int_{S_{m}}\left[G(x, s)-G\left(x, x_{m}\right)\right] \sigma_{m}(s) d s, \quad x_{m} \in D_{m} .
$$

Since $D_{m}$ are small, the choice of $x_{m} \in D$ is not important. If $\left|x-x_{m}\right| \geq d \gg a$, $\left|\zeta_{m}\right|=O\left(a^{-1}\right), 1 \leq m \leq M$, then, as we show, the second sum in (7) is negligible compared with the first, and one can write

$$
u_{M}=u_{0}+\sum_{m=1}^{M} G\left(x, x_{m}\right) Q_{m}+O\left(\frac{a}{d}\right), \quad\left|x-x_{m}\right| \geq d \gg a .
$$

Our aims are: 1) to derive a formula for $\left.Q_{m}, 2\right)$ to pass to the limit $M \rightarrow \infty$ in (8) and to obtain an equation for the effective field $\mathcal{U}(x)$ in the region filled with many small particles, 3) to give a method for creating a "smart " material with a desired refraction coefficient $n^{2}(x)$ by embedding into $D$ many small particles: we give a formula for the number $a^{-1} \mathcal{N}(x) d x$ of particles to be embedded in a volume $d x$ around a point $x$, and for their imepdances $\zeta(x)$, given the desired refraction coefficients $n^{2}(x)$ and $n_{0}^{2}(x)$.

There is a large literature on wave scattering by small particles. The theory was originated by Rayleigh (1871), who studied wave scattering by a single small particle and showed that the main contribution to the scattered field comes from 
the dipole radiation. The smallness of the particle allows one to consider the incident field as a constant field, to solve the static problem, to find the induced dipole moment $P$, and to calculate the corresponding scattered field ([1], §72). Rayleigh did not give formulas for calculating $P$ for particles (small bodies) of arbitrary shapes. This was done by the author (see [4] and references therein). In [4] formulas for electric and magnetic polarizability tensors are derived. In [2] boundary-value problems are studied in domains with boundaries consisting of many small components on which the Dirichlet condition holds, and the operators are elliptic selfadjoint and positive, e.g., Laplace operator. The arguments in [2] do not seem to be applicable to our problem because our operator is not positivedefinite, it may be nonselfadjoint if $n_{0}^{2}(x)$ is a complex-valued function, which corresponds to an absorption, and we use impedance boundary conditions not considered in [2]. It is these coditions that allow us to create materials with arbitrary refraction coefficients. In the case of Neumann boundary condition the Helmholtz operator is considered in [2]. We use some ideas from [5]-[8].

Let $\mathcal{J}_{m}:=\int_{S_{m}} \int_{S_{m}} \frac{d s d t}{|s-t|}, V_{m}=\left|D_{m}\right|$ be the volume of $D_{m},\left|S_{m}\right|$ be the surface area of $S_{m}, \beta_{p j}^{(m)}$ be the magnetic polarizability tensor of $D_{m}$, defined in [4], p. 62, formula (5.62), (see also [3], pp 383-384). Let $\tilde{D} \subset D$ be an arbitrary subdomain of $D$. Assume that

$$
M=O\left(a^{-1}\right), \quad d=O\left(a^{1 / 3}\right), \quad\left|S_{m}\right|=c_{1} a^{2}, \quad \mathcal{J}_{m}=c_{2} a^{3}, \quad V_{m}=c_{3} a^{3} .
$$

These assumptions can be relaxed: the constants $c_{j}, 1 \leq j \leq 3$, may depend on $m$, but we do not go into detail. What is important for our arguments is the order of $M, d, S_{m}, \mathcal{J}_{m}$ and $V_{m}$ as $a \rightarrow 0$. Assumptions (9) hold in Theorem 1. In Theorem 2 the assumptions $M=O\left(a^{-1}\right), d=O\left(a^{1 / 3}\right)$ are no longer valid.

The continuous functions $h(x)$ and $\mathcal{N}(x)$ in Theorem 1 and $\nu(x)$ in Theorem 2 are independent of $a$ and can be chosen a priori. The subdomains $\tilde{D}$ in Theorems 1 and 2 are arbitrary subdomains of $D$ independent of $a$. Let

$$
N_{a}(\tilde{D}):=\sum_{D_{m} \subset \tilde{D}} 1
$$

be the number of particles in $\tilde{D}$. This number grows to infinity as $a \rightarrow 0$, provided that $\int_{\tilde{D}} \mathcal{N}(x) d x>0$.

The function $h(x)$ defines the boundary impedances of the partcles near a point $x \in D$.

The function $\mathcal{N}(x)$ defines the number of small particles in $\tilde{D}$ by the equation (11) in Theorem 1.

The assumption (10) in Theorem 1 means that the small particle $D_{m}$ shrinks to a point $x \in D$, independent of $m$, as $a \rightarrow 0$. For this reason we do not write $x_{m}$ in place of $x$.

Let us now formulate our results. 
Theorem 1 Assume that

$$
\begin{aligned}
& \lim _{a \rightarrow 0} \frac{\zeta_{m} \mathcal{J}_{m}}{4 \pi\left|S_{m}\right|}=h(x), \quad x \in D_{m} \\
& \lim _{a \rightarrow 0} a N_{a}(\tilde{D})=\int_{\tilde{D}} \mathcal{N}(x) d x
\end{aligned}
$$

Then there exists $\lim _{M \rightarrow \infty} u_{M}=\mathcal{U}(x)$, the function $\mathcal{U}(x)$ solves the equation

$$
\begin{aligned}
L \mathcal{U} & :=\left[\nabla^{2}+k^{2}-q(x)\right] \mathcal{U}=0 \quad \text { in } \mathbb{R}^{3}, \quad q(x):=q_{0}(x)+p(x), \\
p(x) & :=\frac{4 \pi c_{1}^{2}}{c_{2}} \frac{\mathcal{N}(x) h(x)}{1+h(x)}
\end{aligned}
$$

where $c_{1}, c_{2}>0$ are the constants from $(9), \mathcal{N}(x)$ is the function from (11), and $h(x)$ is the function from (10). The function $\mathcal{U}=u_{0}+V$, where $V$ satisfies the radiation condition. The scattering amplitude, corresponding to $q(x)$, is:

$$
A(\beta, \alpha)=A_{0}(\beta, \alpha)-\frac{1}{4 \pi} \int_{D} u_{0}(y,-\beta) p(y) \mathcal{U}(y, \alpha) d y
$$

where $A_{0}(\beta, \alpha)$ is the scattering amplitude corresponding to $q_{0}(x)$, and $u_{0}(x, \beta)$ is the scattering solution, corresponding to $q_{0}(x)$. One has:

$$
\mathcal{U}(x, \alpha)=e^{i k \alpha \cdot x}+A(\beta, \alpha) \frac{e^{i k r}}{r}+o\left(\frac{1}{r}\right), \quad r=|x| \rightarrow \infty, \beta:=\frac{x}{r} .
$$

Remark 1 Under the assumptions (9) - (10) one has

$$
\zeta_{m}=\frac{4 \pi c_{1}}{c_{2} a} h(x) \quad \text { as } a \rightarrow 0, \quad x \in D_{m} .
$$

Theorem 2 Assume that $\zeta_{m}=0,1 \leq m \leq M, d \gg a$, and the following limits exist:

$$
\lim _{a \rightarrow 0} \sum_{D_{m} \subset \tilde{D}} V_{m} \beta_{p j}^{(m)}=\int_{\tilde{D}} \beta_{p j}(y) \nu(y) d y, \quad \lim _{a \rightarrow 0} \sum_{D_{m} \subset \tilde{D}} V_{m}=\int_{\tilde{D}} \nu(y) d y,
$$

where $\nu(y) \geq 0$ and $\beta_{p j}(y)$ are continuous functions in $D$ and $\tilde{D}$ is an arbitrary subdomain of $D$. Then there exists the function $\mathcal{U}(x):=\lim _{M \rightarrow \infty} u_{M}(x)$, where $u_{M}(x)$ is defined in $(6)$, and $\mathcal{U}(x)$ solves the equation

$\mathcal{U}(x)=u_{0}(x)+\int_{D} G(x, y) \Delta \mathcal{U}(y) \nu(y) d y-\sum_{p, j=1}^{3} \int_{D} \frac{\partial G(x, y)}{\partial y_{p}} \frac{\partial \mathcal{U}(y)}{\partial y_{j}} \beta_{p j}(y) \nu(y) d y$ 
Example 1 Under the assumptions of Theorem 1, let all the particles be balls of radius $a$. Then $\left|S_{m}\right|=4 \pi a^{2}, \mathcal{J}_{m}=16 \pi^{2} a^{3}, 4 \pi c_{1} / c_{2}=1, p(x)=4 \pi \mathcal{N}(x) h(x)[1+$ $h(x)]^{-1}$ by formula (13), the limit (10) exists iff $\zeta_{m}=h(x) a^{-1}$, where $x \in D_{m}$, and $\mathcal{N}(x)\left|\Delta_{x}\right|=a N\left(\Delta_{x}\right)$, where $\Delta_{x}$ is a small cube centered at $x$ and $N\left(\Delta_{x}\right)$ is the number of small balls of radius $a$ in $\Delta_{x}$. Given an arbitrary continuous function $n^{2}(x), \operatorname{Im} n^{2}(x) \geq 0$, one calculates $q(x)=k^{2}\left[1-n^{2}(x)\right], p(x)=k^{2}\left[n_{0}^{2}(x)-n^{2}(x)\right]$, and then finds the three functions $\mathcal{N}(x) \geq 0, h_{1}(x)$ and $h_{2}(x) \leq 0, h(x):=$ $h_{1}(x)+i h_{2}(x)$, such that

$$
p(x)=4 \pi \mathcal{N}(x) h(x)[1+h(x)]^{-1} .
$$

The function $p(x)=p_{1}(x)+i p_{2}(x)$ satisfies the condition $p_{2}(x) \leq 0$, which means that absorption in the medium is nonnegative. There are many ways to choose $\mathcal{N}(x) \geq 0, p_{1}(x)$ and $p_{2}(x) \leq 0$, so that $(*)$ holds. For example, if $p_{1}(x)>0$, $p_{2} \neq 0$, one may take $h_{1}(x)=0, h_{2}(x)=\frac{p_{1}(x)}{p_{2}(x)}, \mathcal{N}(x)=\frac{p_{1}^{2}(x)+p_{2}^{2}(x)}{4 \pi p_{1}(x)}$. Let us take $\tilde{D}=\Delta_{x}$ and embed in the small cube $\Delta_{x}$ with the side of length $b=10^{-2} \mathrm{~cm}$ the small balls of radius $a=10^{-5} \mathrm{~cm}$ with $d=10^{-3} \mathrm{~cm}$. There will be $\left(\frac{b}{d}\right)^{3}=10^{3}$ small balls in $\Delta_{x}$, the assumption $d \gg a$ is satisfied, condition (11) implies $10^{3}=10^{5} \mathcal{N}(x)\left(10^{-2}\right)^{3}$, i.e., $\mathcal{N}(x)=10^{4}$.

One can create a material with any desirable refraction coefficient $n^{2}(x)$ by embedding into $D$ small balls with the number of balls in a small cube $\Delta_{x}$, centered at a point $x \in D$, calculated by formula (11) and their surface impedances $h(x)$ calculated by formula (10). The functions $\mathcal{N}(x)$ and $h(x)$ are calculated from the given $n^{2}(x)$. We have explained in Example 1 how to calculate $\mathcal{N}(x)$ and $h(x)$ given $n^{2}(x)$.

In Section 2 a brief outline of the derivations of the results is given.

\section{Outline of proof}

Proof of Theorem 1. To derive a formula for the numbers $Q_{m}$ in (8), use the boundary condition (4) and get the following equation:

$$
0=u_{e N}-\zeta_{j} u_{e}+\frac{A_{j} \sigma_{j}-\sigma_{j}}{2}-\zeta_{j} T_{j} \sigma_{j} \quad \text { on } S_{j}, \quad 1 \leq j \leq M,
$$

where $u_{e}$ is the effective field, acting on the $j$-th particle,

$$
\begin{aligned}
T_{j} \sigma_{j} & :=\int_{S_{j}} G(s, t) \sigma_{j}(t) d t, \quad A_{j} \sigma_{j}=2 \int_{S_{j}} \frac{\partial G(s, t)}{\partial N_{s}} \sigma_{j}(t) d t, \\
u_{e}(s) & :=u_{M}(s)-\int_{S_{j}} G(s, t) \sigma_{j}(t) d t .
\end{aligned}
$$

We prove that $G(x, y)=g_{0}(x, y)[1+O(|x-y|)]$ as $|x-y| \rightarrow 0$, where $g_{0}:=$ $(4 \pi|x-y|)^{-1}$. Thus, we can replace $G$ by $g_{0}$ in $T_{j}$ and in $A_{j}$. Then we use 
the relation $\int_{S_{j}} A_{j} \sigma_{j} d s=-\int_{S_{j}} \sigma_{j} d s$, see [4], p.96, formula (7.21). Integrating (19) over $S_{j}$ we derive after some calculations (see [4], p.97, formula (7.26)), the following equation:

$$
Q_{j}=-\frac{\zeta_{j}\left|S_{j}\right|}{1+\zeta_{j} \mathcal{J}_{j}\left(4 \pi\left|S_{j}\right|\right)^{-1}} u_{e}\left(x_{j}\right)
$$

This and (8) imply

$$
u_{M}=u_{0}-\sum_{m=1}^{M} G\left(x, x_{m}\right) \frac{4 \pi c_{1}^{2} h\left(x_{m}\right) a}{c_{2}\left[1+h\left(x_{m}\right)\right]} u_{e}\left(x_{m}\right) .
$$

To pass to the limit $M \rightarrow \infty$, i.e., $a \rightarrow 0$, in (22), we use (10) and (11) and get

$$
\mathcal{U}(x)=u_{0}(x)-\int_{D} G(x, y) p(y) \mathcal{U}(y) d y
$$

Applying the operator $L_{0}$ to (23) and using the equation $L_{0} G=-\delta(x-y)$, one gets equation (12), and formula (13) for $p(x)$.

Clearly $\mathcal{U}$ satisfies the radiation condition (15). Formula (14) follows from (23) and the author's lemma (see [3], p. 257, formula (5.17)). In the limit $a \rightarrow 0$ there is no difference between $\lim _{a \rightarrow 0} u_{e}(x)$ and $\lim _{a \rightarrow 0} u_{M}(x)$.

Outline of the proof of Theorem 2. The principal novelty from the physical viewpoint is that if $\zeta_{m}=0 \forall m$, then the second sum in (7) is of the same order of magnitude as the first (it can be even larger than the first). Therefore, now the second term in (7) cannot be neglected, in contrast to the case considered in Theorem 1. In place of equation (19), now one gets:

$$
0=u_{e N}+\frac{A_{j} \sigma_{j}-\sigma_{j}}{2}
$$

Integrating this equation over $S_{j}$, one gets

$$
Q_{j}=\int_{S_{j}} u_{e N} d x=\int_{D_{j}} \Delta u_{e} d x \simeq \Delta u_{e}\left(x_{j}\right) V_{j}
$$

Thus, now $Q_{j}=O\left(k^{2} a^{3}\right)$, while under the assumptions of Theorem 1 we had $Q_{j}=O(a)$. Since $k a \ll 1$, the quantity $Q_{j}$ is now much smaller than in Theorem 1. Let us show that the integral

$$
I_{j}:=\int_{S_{j}}\left[G(x, s)-G\left(x, x_{j}\right)\right] \sigma_{j}(s) d s
$$

is of the same order of magnitude as $Q_{j}$. 
We have $G(x, s)-G\left(x, x_{m}\right)=\left.\nabla_{y} G(x, y)\right|_{y=\tilde{y}} \cdot\left(s-x_{m}\right)$, where $\tilde{y}$ is some point of the segment $\left(x_{m}, s\right)$. If $\left|x-x_{j}\right| \geq d \gg a$, and $a$ is very small, then $\nabla_{y} G(x, \tilde{y}) \simeq \nabla_{y} G\left(x, x_{j}\right)$. Thus,

$$
I_{j}=\frac{\partial G\left(x, x_{j}\right)}{\partial y_{p}} \int_{S_{j}}\left(s-x_{j}\right)_{p} \sigma_{j}(s) d s,
$$

where over the repeated index $p$ one sums up, and $\left(s-x_{j}\right)_{p}$ is the $p$-th coordinate of the vector $s-x_{j}$. The integral in $I_{j}$ is calculated using formulas (5.61), and (5.62), in [4], p.62. The result is:

$$
\int_{S_{j}}\left(s-x_{j}\right)_{p} \sigma_{j}(s) d s=-V_{j} \frac{\partial u_{e}\left(x_{j}\right)}{\partial y_{\ell}} \beta_{p \ell}^{(j)},
$$

where $\beta_{p \ell}^{(j)}$ is the magnetic polarizability tensor defined in [4], p. $62, V_{j}$ is the volume of $D_{j}$, and over the repeated index $\ell$ (but not over $j$ ) one sums up. The quantity $I_{j}=O\left(k^{2} a^{3}\right)$, i.e., it is of the same order as $Q_{j}$. Indeed, $V_{j}=O\left(a^{3}\right)$, and two differentiations with respect to $y_{p}$ and $y_{\ell}$ bring the factor $k^{2}$. Thus

$$
u_{M}(x)=u_{0}(x)+\sum_{m=1}^{M} G\left(x, x_{m}\right) \Delta u_{e}\left(x_{j}\right) V_{j}-\sum_{m=1}^{M} \sum_{p, \ell=1}^{3} \frac{\partial G\left(x, x_{m}\right)}{\partial y_{p}} V_{m} \frac{\partial u_{e}\left(x_{m}\right)}{\partial y_{\ell}} \beta_{p \ell}^{(j)} .
$$

Passing to the limit $M \rightarrow \infty, a \rightarrow 0$, and using (7), one obtains equation (18). The existence of the second limit (17) is compatible with the assumption $d \gg a$ only if $\nu(y)$ is very small. Indeed, the second equation (17) implies that $\nu(y)=O\left(a^{3} d^{-3}\right)$.

If $\nu(y)$ and $\nabla \nu(y)$ are very small, then the first approximation to the solution of equation (18) is given by the formula

$$
\mathcal{U}(x)=u_{0}(x)+\int_{D} G(x, y) \Delta u_{0} \nu(y) d y-\sum_{p, j=1}^{3} \int_{D} \frac{\partial G(x, y)}{\partial y_{p}} \frac{\partial u_{0}(y)}{\partial y_{j}} \beta_{p j}(y) \nu(y) d y
$$

In general, equation (18) is not local, it is an integral-differential equation. If one assumes that $\left.\nu(y)\right|_{S}=0$, then one can integrate by parts the last integral in (18) and get an equation from which a differential equation can be derived:

$$
\mathcal{U}(x)=u_{0}(x)+\int_{D} G(x, y)\left[\nu(y) \Delta \mathcal{U}(y)+\sum_{p, j=1}^{3} \frac{\partial}{\partial y_{p}}\left(\frac{\partial \mathcal{U}(y)}{\partial y_{j}} \beta_{p j}(y) \nu(y)\right)\right] d y .
$$

Applying the operator $L_{0}$ to (28) one gets a differential equation:

$$
L_{0} \mathcal{U}+\nu(y) \Delta \mathcal{U}+\sum_{p, j=1}^{3} \frac{\partial}{\partial y_{p}}\left(\frac{\partial \mathcal{U}(y)}{\partial y_{j}} \beta_{p j}(y) \nu(y)\right)=0
$$

In [9] one finds detailed proofs. 


\section{References}

[1] Landau, L.D., Lifshitz, E.M., Electrodynamics of continuous media, Pergamon Press, Oxford, 1960.

[2] Marchenko, V., Khruslov, E., Boundary-value problems in domains with fine-grained boundary, Naukova Dumka, Kiev, 1974 (Russian).

[3] Ramm, A.G., Inverse problems, Springer, New York, 2005.

[4] Ramm, A.G., Wave scattering by small bodies of arbitrary shapes, World Sci. Publ., Singapore, 2005.

[5] Ramm, A.G., Equations for the self-consistent field in random medium, Phys.Lett. A, 312, N3-4, (2003), 256-261.

[6] Ramm, A.G., Electromagnetic wave scattering by many small particles, Phys. Lett. A, 360, N6, (2007), 735-741.

[7] Ramm, A.G., Distribution of particles which produces a "smart" material, Jour. Stat. Phys., 127, N5, (2007), 915-934.

[8] Ramm, A.G., Many-body wave scattering by small bodies, J. Math. Phys., 48, N2, 023512, (2007).

[9] Ramm, A.G., J. Math. Phys, 48, N10, 103511 (2007) 